\title{
'Saco vazio não para em pé': Programa Bolsa Família e mortalidade por desnutrição
}

\author{
'An empty bag cannot stand upright': The Bolsa Familia Program and \\ mortality caused by malnutrition
}

Rafael dos Santos', Carla Garcia Bottega²

DOI: 10.1590/0103-1104201912216

RESUMO As condições de saúde resultam de fatores socioeconômicos, sobretudo aquelas doenças de causas evitáveis, como a desnutrição em crianças nos seus primeiros anos de vida. Assim, o objetivo deste estudo foi investigar a relação entre a mortalidade na infância por desnutrição e as ações em saúde do Programa Bolsa Família, comparando dados nacionais com dados do Rio Grande do Sul. Foram correlacionados, por meio do método de Pearson, dados referentes ao Programa Bolsa Família (número de famílias beneficiárias, condicionalidades em saúde colocadas pelo Programa e valores transferidos às famílias beneficiárias) com as taxas de mortalidade por desnutrição em crianças menores de cinco anos entre os anos 2005 e 2014 . Os dados, cuja coleta foi realizada entre agosto e setembro de 2016, são originários da Matriz de Informação Social (MI Social), das bases do Sistema de Informações sobre Nascidos Vivos e do Sistema de Informações de Mortalidade. Os resultados demonstraram que o Bolsa Família atua por meio da transferência de renda, pois impacta no estado nutricional da criança, e exaltaram o caráter intersetorial do Programa, possibilitando melhores condições de cidadania aos seus beneficiários, por meio da ampliação do acesso aos serviços públicos e da participação em ações de promoção e prevenção em saúde.

PALAVRAS-CHAVE Mortalidade da criança. Desnutrição. Promoção da saúde. Colaboração intersetorial.

1 Universidade Federal do Rio Grande do Sul (UFRGS) - Porto Alegre (RS), Brasil. d.santosrafael@gmail.com

2 Universidade Estadual do Rio Grande do Sul (UERGS)

- Porto Alegre (RS), Brasil.
ABSTRACT The health of a person is the result of socioeconomic factors, especially those pertaining to preventable conditions, such as malnutrition in early childhood. Thus, this study investigated the relation between child mortality caused by malnutrition and the health measures of the Bolsa Famillia (Family Allowance) program in Brazil, comparing nationwide data with data from the state of Rio Grande do Sul. The Pearson correlation coefficient was used to analyze data from Bolsa Familia (the number of families benefitting from the program, the health conditions it requires for eligibility, and the amount of money transferred to the families) in relation to malnutrition mortality rates among children under five, from 2005 to 2014. The data, collected between August and September 2016, were obtained from the Social Information Center (Matriz de Informação Social) of the Live Birth Information System (Sistema de Informações Sobre Nascidos Vivos) and the Mortality Information System (Sistema de Informações de Mortalidade) of Brazil. The results demonstrate that 
Bolsa Familia functions by transferring income, which positively affects the nutrition of children, and that its intersectionality allows for better citizenship conditions for families, by broadening access to public services and participating in health promotion and disease prevention programs.

KEYWORDS Child mortality. Malnutrition. Health promotion. Intersectoral collaboration.

\section{Introdução}

Conforme Monteiro ${ }^{\mathbf{1}}$, a desnutrição decorre da insuficiência do aporte alimentar em energia e nutrientes, ou então, do aproveitamento biológico inadequado dos alimentos ingeridos. Na infância, a desnutrição, refletida por indicadores antropométricos do estado nutricional configura-se em um dos maiores problemas de saúde enfrentados por países em desenvolvimento, pois responde por cerca de 3,5 milhões de óbitos infantis no mundo ${ }^{2,3}$. Segundo Caulfield et al. ${ }^{4}$, a desnutrição infantil compreende atraso de crescimento, ou seja, baixa estatura para a idade; subnutrição, baixo peso para a altura com indicação de perda de peso aguda e baixo peso para a idade. $\mathrm{Na}$ maioria dos casos, tem origem em fatores socioeconômicos, visto que crianças provenientes de famílias pobres ou extremamente pobres possuem menores condições de receberem uma alimentação qualificada, em termos de ingestão de nutrientes, ou em quantidade suficiente para seu melhor desenvolvimento ${ }^{5}$.

Black et al. ${ }^{6}$ e Caulfield et al. ${ }^{7}$ apontam para a relação entre a desnutrição e a sobrevivência da criança, uma vez que o aumento do nível de desnutrição infantil faz crescer o risco de óbito, em particular, por infecções como diarreia e sarampo. Ramachandran et al. ${ }^{\mathbf{8}}$ atentam para o ciclo vicioso existente entre essas duas causas, de modo que a desnutrição debilita as funções imunitárias do organismo e, consequentemente, aumenta o risco de infecções, da mesma forma que as doenças infecciosas prejudicam o estado nutricional da criança.
A Rede Interagencial de Informação para Saúde (Ripsa) ${ }^{9}$ define a Taxa de Mortalidade em Menores de Cinco Anos (TMM5), como o número de óbitos destes indivíduos, por mil nascidos vivos, na população residente em determinado território ou espaço geográfico, no ano considerado. A TMM5 é capaz de estimar o risco de morte dos nascidos vivos durante os cinco primeiros anos de vida e expressar, genericamente, o desenvolvimento socioeconômico e a infraestrutura ambiental insuficiente, que condicionam a desnutrição infantil e outras doenças a ela associadas. Além disso, a interpretação dos coeficientes de mortalidade nesse grupo etário contribui para diagnosticar a situação do acesso aos serviços de saúde e a qualidade dos recursos disponíveis para atender à saúde materno-infantil ${ }^{9}$.

Criado por meio da Lei Federal $\mathrm{n}^{\circ} 10.836$, de 9 de janeiro de $2004^{10}$, e regulamentado pelo Decreto no $5.209 / 2004^{11}$, o Programa Bolsa Família (PBF) surgiu com o intuito de unificar a gestão e a execução das ações de outros programas de transferência de renda do Governo Federal. Dessa forma, os Programas Bolsa Escola, Bolsa Alimentação, Auxílio Gás e Cartão Alimentação, antes vinculados a diferentes órgãos gestores, foram integrados em único Programa de Transferência de Renda Condicionada (PTRC) e passaram a ser administrados pelo Ministério do Desenvolvimento Social. Em relação a seus beneficiários, o Bolsa Família propõe: (I) promover o acesso à rede de serviços públicos, especialmente de saúde, educação e assistência social; (II) combater a fome e promover segurança alimentar e 
nutricional; (III) estimular a emancipação sustentada das famílias que vivem em situação de pobreza e extrema pobreza; (IV) combater a pobreza e (V) promover a intersetorialidade, a complementaridade e a sinergia das ações sociais do Poder Público"1. Essas metas qualificam o PBF como uma política adjuvante às ações de segurança alimentar e à melhora deste indicador em virtude da sua relação com aspectos socioeconômicos.

O Fundo das Nações Unidas para a Infância (Unicef) indica que as iniquidades socioeconômicas se manifestam nas condições de saúde da população, especialmente em crianças nos primeiros anos de vida, por meio do efeito cumulativo da pobreza 9 . Assim, por entender que os PTRC podem se configurar em um mecanismo catalisador do desenvolvimento socioeconômico e contribuir para a melhora dos níveis de saúde da população beneficiada por eles, este trabalho tem por objetivo investigar como as ações em saúde do Bolsa Família relacionam-se com as TMM5 por desnutrição no Brasil e no estado do Rio Grande do Sul (RS). A comparação dessa unidade da federação com dados nacionais é justificada pela situação de Segurança Alimentar e Nutricional (SAN) superior do RS em comparação à média nacional ${ }^{12}, 80,8 \%$ contra $69,8 \%$, permitindo avaliar a efetividade do PBF em um contexto de maior acesso à alimentação.

\section{Material e métodos}

Esta é uma pesquisa avaliativa, baseada em dados secundários obtidos no Sistema de Informações sobre Nascidos Vivos (Sinasc) e no Sistema de Informações de Mortalidade (SIM). Esses dados consistem em estatísticas vitais referentes ao número de nascidos vivos por residência da mãe e ao número de óbitos, por local de residência, entre crianças com idade menor de cinco anos por causas relacionadas com a desnutrição, elencadas pela Classificação Estatística Internacional de Doenças e Problemas Relacionados com a Saúde (CID 10) por meio dos códigos E 40 a $\mathrm{E} 46$.

As TMM5 foram calculadas adaptando o método usual, indicado pela Ripsa, em razão deste trabalho, focando somente nas mortes por desnutrição. Logo, as taxas de mortalidade são obtidas pelo método direto, dividindo o número de óbitos por causas relacionadas com desnutrição por mil nascidos vivos.

As informações sobre a cobertura e o número de beneficiários do $\mathrm{PBF}$, bem como aquelas referentes ao acompanhamento das condicionalidades em saúde, foram obtidas a partir dos dados divulgados pela Secretaria de Avaliação e Gestão da Informação (Sagi) do Ministério do Desenvolvimento Social por meio da Matriz de Informação Social (MI Social). Todos os dados relacionados com a natalidade e com a mortalidade, assim como aqueles que dizem respeito ao PBF, foram coletados entre agosto e setembro de 2016.

Foram verificadas as correlações existentes entre as variáveis número médio anual de famílias acompanhadas pelas ações de saúde do PBF e as TMM5 por desnutrição no Brasil e no RS; correlação que é definida por Stevenson ${ }^{\mathbf{1 3}}$ como a medida da força ou do grau de relacionamento entre duas variáveis. Apurou-se também o grau de relacionamento entre a cobertura da Estratégia Saúde da Família (ESF) com as TMM5 por desnutrição e com a cobertura do PBF sob forma de verificar se este cumpre com a proposta de possibilitar o acesso à rede de serviços básicos de saúde.

A forma utilizada para verificar a correlação entre as variáveis foi coeficiente $r$ de Pearson, definido por Figueiredo Filho e Silva Júnior ${ }^{14(118)}$ como "[...] uma medida de associação linear entre variáveis", que sintetiza o grau de relacionamento entre elas por meio de um fator que varia entre $-1,00$ e $+1,00$.

Assim, segundo Stevenson ${ }^{\mathbf{1 3}}$, quando o $r$ de Pearson for positivo, ocorre que as duas variáveis se relacionam de forma 
diretamente proporcional, ou seja, à medida que um valor cresce, o valor da variável dependente tende a aumentar. No caso de o coeficiente de Pearson apresentar um valor negativo, significa dizer que o relacionamento entre as variáveis é inversamente proporcional. Sendo assim, enquanto uma variável aumenta, a outra tende a diminuir.

Em um coeficiente de Pearson igual ou bem próximo a 0,00 , indica-se a ausência de relação entre as variáveis. O coeficiente de determinação, definido, conforme Stevenson ${ }^{13}$, como uma medida descritiva da proporção da variação de $\mathrm{Y}$ que pode ser explicada por variações em $X$, foi outra medida utilizada para apurar quanto, em termos percentuais, o comportamento de uma variável poderia ser determinado pelo comportamento de outra.

\section{Resultados e discussão}

Os primeiros resultados referem-se à correlação entre o número de famílias acompanhadas pelas ações de saúde do PBF e as TMM5 por desnutrição. Em nível nacional, observa-se uma correlação negativa muita alta $(r=-0,98)$ entre o número de famílias acompanhadas pelas ações em saúde do PBF e as TMM5 por desnutrição. Esse resultado aponta que, quanto maior o número de famílias acompanhadas, menores as taxas de mortalidade por desnutrição. Observa-se, também, que o coeficiente de determinação $\left(\mathrm{r}^{2}\right)$ aponta que $95,21 \%$ da variação da taxa de mortalidade está associada ao aumento da população de famílias acompanhadas pelas ações em saúde do PBF (gráfico 1).

Gráfico 1. Correlação entre o número de famílias acompanhadas pelas ações em saúde do PBF e as TMM5 por desnutrição; Brasil, 2005-2014

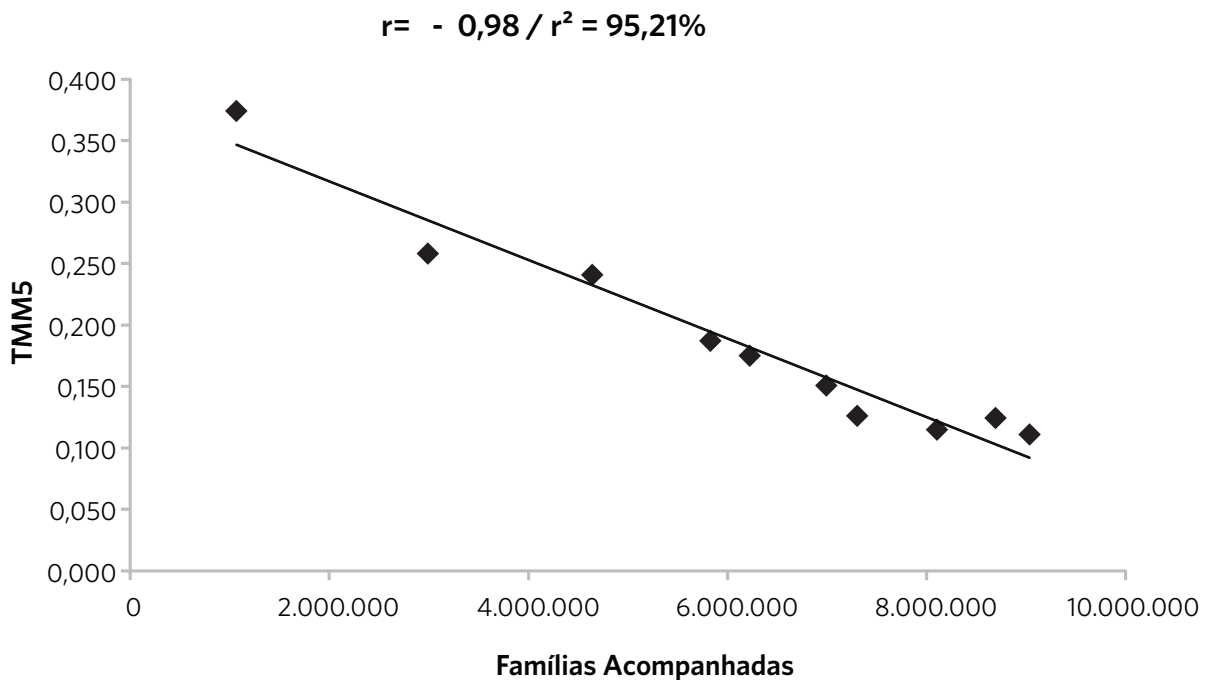

Fonte: Elaboração própria. Baseado em Sagi e Datasus.

Esse resultado explica-se pelo caráter intersetorial proposto pelo PBF, que opera de maneira associada a uma perspectiva de garantia de direitos sociais básicos e efetivação das ofertas universais à população em situação de desigualdade. Desta forma, Ximenes e Agatte ${ }^{15(13)}$ corroboram este pensamento quando sugerem que a: 
[...] sinergia gerada pela oferta simultânea do benefício monetário e dos serviços sociais de educação, saúde e assistência social potencializa a capacidade de desenvolvimento das famílias, contribuindo para sua melhor inserção na dinâmica da vida social, comunitária e produtiva.

Da Silva e Volpato ${ }^{16(16)}$ compartilham da mesma visão na medida em que afirmam "Uma importante parceria é entre a saúde e a assistência social [...]", pois, nessa parceria, é possível aos profissionais realizarem intervenções que façam diferença na vida dessas pessoas, principalmente em relação a seus direitos, muitas vezes não reconhecidos. Cabe ressaltar que o aumento do número de famílias acompanhadas resulta também do entendimento de que PTRC servem como um mecanismo de grande valia à resolução de entraves enfrentados pelas populações mais pobres dos países em desenvolvimento para acessarem os serviços de saúde.

Nesse contexto, destaca-se a importância das condicionalidades do PBF que, conforme Silva ${ }^{17(56)}$, constituem-se em mecanismos contributivos ao acesso e ampliação de direitos sociais básicos e incentivam a demanda,

[...] responsabilizando o Estado na oferta de serviços públicos e às famílias beneficiárias no cumprimento das condicionalidades fixadas pelo Programa [...].

Seu acompanhamento é realizado de forma conjunta e articulada com os registros do Ministério da Saúde, da Educação e do Desenvolvimento Social e Combate à
Fome ${ }^{10}$. As condicionalidades em saúde do PBF incluem ações divididas em focos de atuação, cabendo aos beneficiários do programa o cumprimento de contrapartidas, como: acompanhamento pré-natal; participação em atividades educativas, para gestantes e nutrizes, respectivamente; acompanhamento do crescimento e desenvolvimento das crianças de 0 a 7 anos e cumprimento do calendário de vacinas.

Consequentemente, essas contrapartidas podem se configurar em medidas eficazes na promoção e no incentivo ao uso dos serviços de saúde. No entanto, a fim de que esses resultados se tornem efetivos, é necessária a combinação com intervenções do Estado para que esses efeitos sejam produzidos e ou maximizados ${ }^{18}$, logo, a justificativa para a inclusão do PBF na relação de indicadores do Pacto pela Saúde, o qual responsabiliza o Sistema Único de Saúde (SUS) pelo acompanhamento das ações de nutrição e saúde das famílias beneficiárias e inclui, entre as metas de fortalecimento da atenção básica, o monitoramento semestral de, ao menos, $80 \%$ das famílias com perfil de saúde beneficiárias do PBF 19 .

Convém salientar a relevância da ESF para a concretização dos objetivos do PBF no que tange à promoção do acesso aos serviços básicos em saúde e à segurança alimentar e nutricional, preconizados no art. $4^{\circ}$ do Decreto Federal $n^{0} 5.209 / 2004^{10}$. Na promoção do acesso, essa relação entre a ESF e o PBF é percebida no crescimento conjunto e correlacionado entre a cobertura da ESF, estimada por meio da média anual da população coberta e pelo número de famílias assistidas pelo Programa (tabela 1). 
Tabela 1. Coeficiente Pearson e coeficiente de determinação, Brasil, 2005-2014

\begin{tabular}{lrr}
\hline Relação & Pearson $(r)$ & Coeficiente de determinação $\left(r^{2}\right)$ \\
\hline ESF x PBF & 0,94 & $89,11 \%$ \\
ESF x Acompanhamento & 0,97 & $94,77 \%$ \\
ESF X TMM5 & $-0,94$ & $87,57 \%$ \\
PBF x Acompanhamento & 0,94 & $89,02 \%$ \\
PBF x TMM5 & $-0,94$ & $88,60 \%$ \\
\hline
\end{tabular}

Fonte: Elaboração própria.

Os coeficientes de Pearson (r), expostos na tabela 1, apontam uma correlação positiva muito alta entre elas, ratificando o argumento apresentado anteriormente de que o PBF estaria associado com a ampliação do acesso aos serviços de saúde, neste caso, representado pela cobertura da ESF. Assim, à medida que aumentam as coberturas da ESF e do PBF, bem como o número de famílias acompanhadas pelo programa, as taxas de mortalidade por causas ligadas à desnutrição tendem a diminuir, conforme indica o coeficiente $r$ entre essa variável e as demais.

É possível observar também que tanto as coberturas do PBF quanto as da ESF apresentam uma intensidade de correlação negativa muito alta com as taxas de mortalidade por desnutrição. Nota-se ainda que a correlação entre a cobertura da ESF e o acompanhamento das condicionalidades do PBF apresenta uma intensidade negativa muito alta e maior, inclusive, do que o coeficiente de Pearson obtido por meio do relacionamento entre o PBF e o acompanhamento das ações em saúde do programa. Logo, deduz-se que a efetividade do Programa sobre os resultados em saúde, neste caso, representados pelas taxas de mortalidade na infância por desnutrição, dependeria mais do acesso aos serviços de saúde - em particular, à atenção básica - assim como da qualidade ofertada por esses serviços ${ }^{\mathbf{1 8 , 2 0}}$. Ranganathan e Lagarde ${ }^{18}$ corroboram essa afirmativa ao apontarem que o efeito do PBF nas TMM5 por desnutrição é resultado do aumento propiciado pelos programas de transferência de renda condicional aos serviços de prevenção. Dessa forma, a correlação entre o PBF e as TMM5 resulta da interação entre as ações do Programa, sobretudo aquelas relacionadas com a saúde e com o SUS.

No estado do RS, o primeiro resultado obtido aponta que, assim como os resultados demonstrados na análise nacional, a correlação entre o acompanhamento das condicionalidades e as taxas de mortalidade no estado apresenta um grau de intensidade negativa muito alta ${ }^{21}(-0,87)$ e que $75,62 \%\left(\mathrm{r}^{2}\right)$ do comportamento das TMM5 por desnutrição ao longo da série histórica é determinado pelo número de famílias acompanhadas pelas ações em saúde do PBF (gráfico 2). No entanto, esses índices, quando comparados aos apresentados em nível nacional $\left(r=-0,98\right.$ e $\mathrm{r}^{2}=$ $95,21 \%)$, mostram-se inferiores. 
Gráfico 2. Correlação entre o número de famílias acompanhadas pelas ações em saúde do PBF e as TMM5 por desnutrição; Rio Grande do Sul, 2005-2014

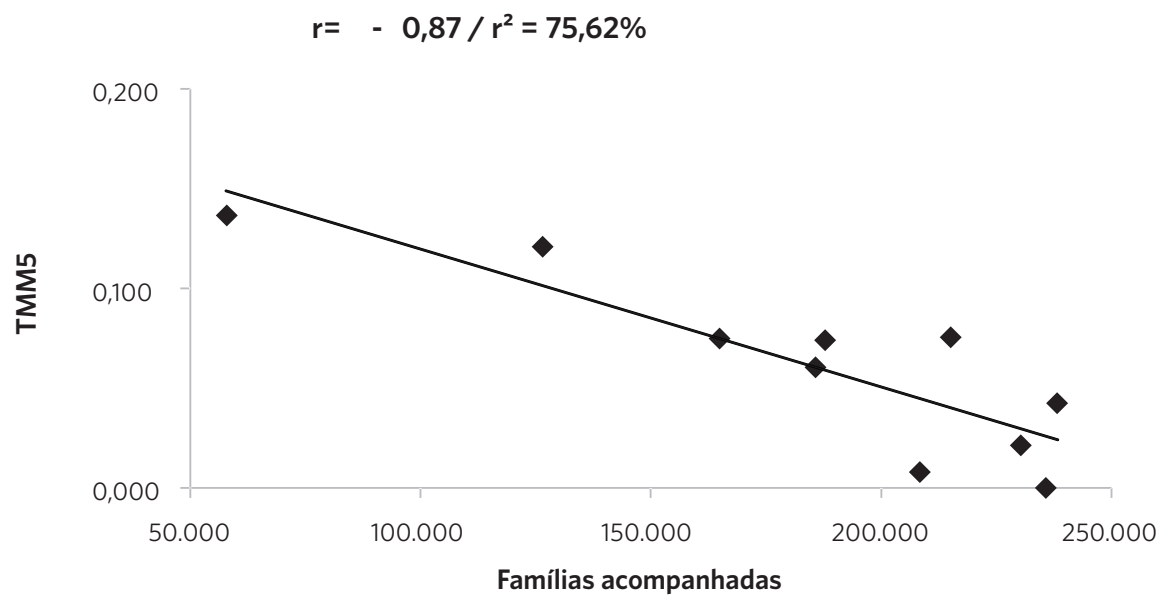

Fonte: Elaboração própria. Baseado em Sagi e Datasus.

Observando os mesmos indicadores de análise explorados no âmbito nacional, quais sejam eles: a cobertura do PBF e da ESF, além do acompanhamento das condicionalidades em saúde do Programa no RS, bem como o grau de correlação existente entre essas variáveis (tabela 2), obteve-se que os resultados demonstram um grau de relacionamento de intensidade negativa moderada entre o PBF e as taxas de mortalidade na infância por desnutrição, assim como entre a cobertura do programa e o acompanhamento de suas ações em saúde $(r=-0,54$ e $r=-0,50$, respectivamente $)$.

Tabela 2. Coeficiente Pearson e coeficiente de determinação, Rio Grande do Sul, 2005-2014

\begin{tabular}{lrr}
\hline Relação & Pearson $(r)$ & Coeficiente de determinação $\left(r^{2}\right)$ \\
\hline PBF $\times$ TMM5 & $-0,54$ & $28,89 \%$ \\
PBF $\times$ Acompanhamento & $-0,50$ & $25,50 \%$ \\
ESF $\times$ PBF & 0,47 & $21,95 \%$ \\
ESF $\times$ Acompanhamento & 0,88 & $77,24 \%$ \\
ESF X TMM5 & $-0,77$ & $59,75 \%$ \\
\hline
\end{tabular}

Fonte: Elaboração própria. 
Considerando a regressão $\left(\mathrm{r}^{2}\right)$ obtida ao relacionar o número de beneficiários com as variáveis taxas de mortalidade por desnutrição $\left(\mathrm{r}^{2}=28,89 \%\right)$ e acompanhamento das ações em saúde do $\mathrm{PBF}\left(\mathrm{r}^{2}=25,50 \%\right)$, não é possível associar as TMM5 por desnutrição e o acompanhamento das ações em saúde com o Bolsa Família. O mesmo ocorre na correlação entre a abrangência da ESF e a cobertura do PBF no estado ( $r=0,47$ e $\left.r^{2}=21,95 \%\right)$, que, diferentemente dos resultados obtidos em nível nacional, não apresentam uma influência significativa do Programa no acesso à saúde para as famílias beneficiárias. Uma hipótese para essas diferenças em relação aos resultados aferidos no Brasil é o desempenho regularmente superior do Programa Saúde da Família (PSF), na região Sul22, principalmente em contextos de maior iniquidade social e de saúde, pois a mortalidade na infância estaria mais influenciada pela efetividade da Atenção Primária à Saúde (APS) ofertada nesse território. Os resultados que serão apresentados a seguir ratificam essa conclusão.

A ESF demonstra uma maior associação com as TMM5 por desnutrição, conforme o grau de correlação entre essas variáveis $(\mathrm{r}$ $=-0,77)$, assim como seu coeficiente de determinação $\left(r^{2}=59,75 \%\right)$. A ESF pode ser considerada também como um determinante importante na variação do número de famílias acompanhadas pelas ações em saúde do PBF, tendo em vista a intensidade positiva muito alta apresentada nessa correlação $(r=0,88)$, e seu coeficiente de determinação, que indica um percentual de $77,24 \%$.

Esses índices reforçam a importância da intersetorialidade para a melhor execução das ações propostas pelo Programa, sobretudo aquelas concernentes à Saúde Pública. As correlações apresentadas neste estudo apontam que o Programa pode ter interferência sobre o comportamento das TMM5 por desnutrição ao longo da série histórica analisada, embora o modelo estatístico utilizado não permita que se infira uma relação de causa-efeito ${ }^{23}$. No entanto, como pode-se observar, há a ocorrência de uma associação entre as ações do PBF, sobretudo aquelas relacionadas com a saúde, tanto no âmbito nacional quanto estadual. Possivelmente, essa relação justifica-se pela atuação do Programa conjuntamente com a APS sobre os determinantes que agem sobre a sobrevivência da criança.

Rasella $^{20}$ sintetiza e sugere que a atuação da transferência de renda condicional sobre a sobrevivência da criança ocorre em três domínios (figura 1). 
Figura 1. Modelo teórico dos efeitos dos programas de transferências condicionadas de renda e da Atenção Primária à Saúde sobre a sobrevivência da criança

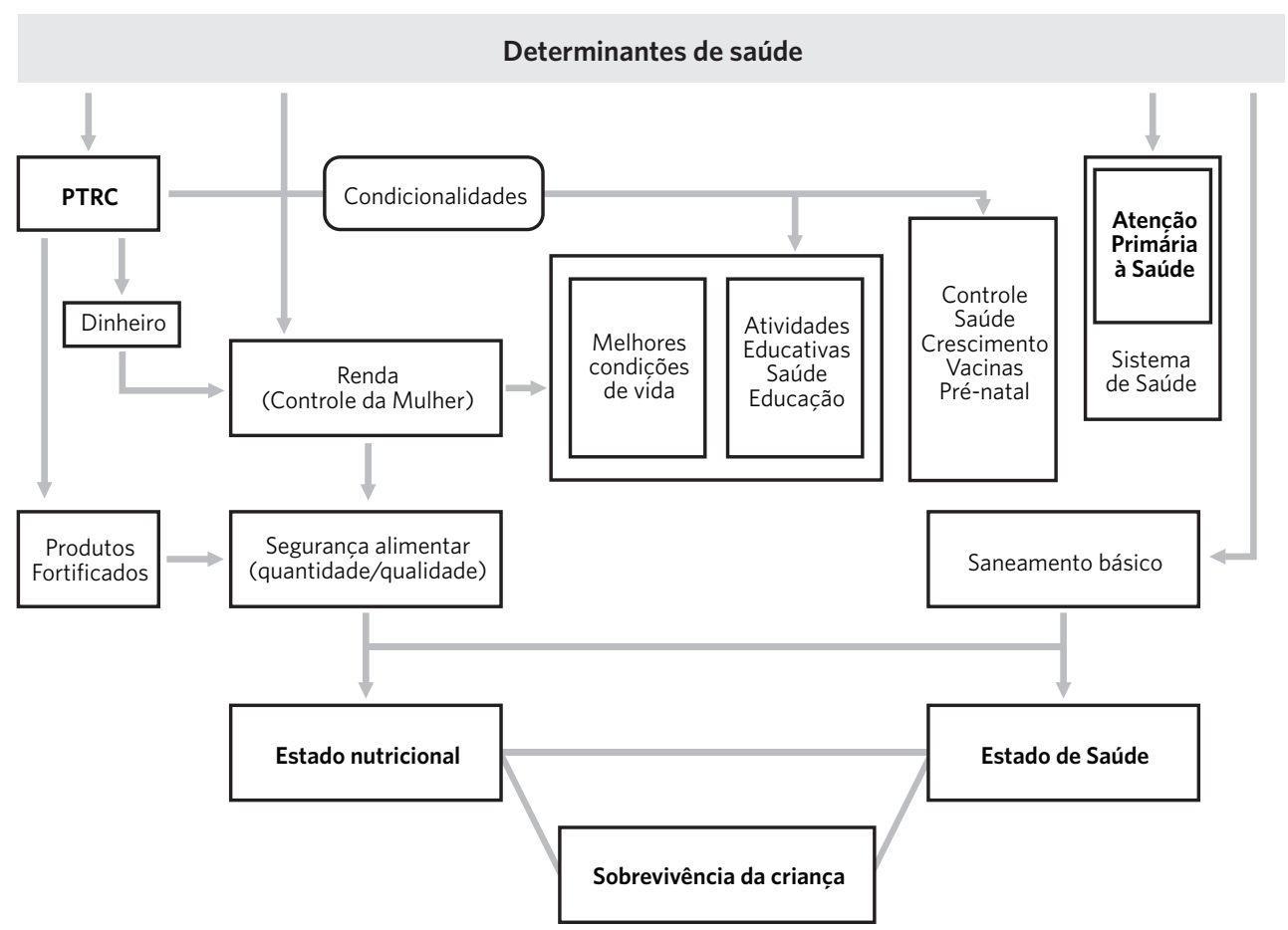

Fonte: Elaboração própria, baseado em Rasella20

O primeiro deles, por óbvio, é mediante o valor monetário transferido às famílias beneficiárias, o qual possibilitaria uma melhora em suas condições de vida, principalmente em relação à aquisição de produtos de higiene e farmacêuticos. Outro aspecto diz respeito à segurança alimentar, sendo este um produto fortificado pela renda também e que impacta no estado nutricional da criança. Por fim, encontram-se as condicionalidades colocadas pelo PBF aos beneficiários, que servem como elo entre a assistência social e o sistema de saúde. Sua atuação ocorre por intermédio de atividades de promoção e educação em saúde e nutrição, bem como dos controles de vacinação, crescimento das crianças e pré-natal das gestantes. Essas medidas agem sobre o estado de saúde da população de beneficiários e conjuntamente com a melhora do estado nutricional, propiciado pelo programa de transferência de renda, e têm efeito na sobrevivência da criança.

\section{Considerações finais}

Este artigo não teve a pretensão de explicar o comportamento das taxas de mortalidade na infância por desnutrição por meio de uma relação de causa-efeito com o PBF, pois entende que o modelo estatístico adotado na análise serve, tão somente, para verificar a ocorrência de uma associação entre duas variáveis. Dessa forma, procurou-se, com este estudo, investigar a correlação entre o PBF e as TMM5 por desnutrição no Brasil e no estado do RS sob a hipótese de que o Bolsa Família transcenderia a forma de uma política assistencialista e propiciaria, por intermédio de seus objetivos, uma situação de equidade às populações mais pobres em todos os aspectos, criando, assim, melhores condições de cidadania a elas por meio do acesso aos seus direitos sociais; o que, consequentemente, geraria resultados positivos sobre a saúde dessas pessoas. 
Assim, os resultados obtidos na pesquisa refutam o caráter meramente assistencialista imputado ao Programa, pois demonstraram um grau de correlação negativo alto entre as variáveis renda transferida, acompanhamento das condicionalidades em saúde e TMM5 por desnutrição, tanto no Brasil quanto no RS. Logo, as informações obtidas com o tratamento desses dados sugerem que o PBF pode produzir resultados em saúde, em particular, sobre a mortalidade na infância, porém, como fora dito anteriormente, não se pode afirmar que seja o causador de determinado comportamento, mas é um dos fatores de sua composição.

Em relação à ampliação do acesso à saúde, procurou-se correlacionar, em paralelo, a cobertura da ESF e do PBF, ainda que não estivesse proposto entre os objetivos desse trabalho, como forma de averiguar se o aumento da cobertura dessas duas políticas públicas tivesse alguma associação. Verificou-se que a ESF poderia, sim, ter influência sobre as taxas de mortalidade na infância por desnutrição, seja por conta de sua alta correlação com o Programa, no caso do Brasil, seja pelo acompanhamento de suas condicionalidades em saúde, ou então, de maneira direta, em razão de os seus objetivos envolver a promoção e a prevenção em saúde, como ocorrera no estado do RS.

Pôde-se constatar, com este estudo, que o Bolsa Família sozinho não é capaz de produzir algum resultado positivo sobre qualquer agravo em saúde; nem ao menos em uma situação que esteja tão ligada a aspectos econômicos e sociais, como a mortalidade na infância. O contrário também se faz verdadeiro, pois, por mais eficiente e eficaz que a saúde pública seja, o desenvolvimento social e o combate às iniquidades do sistema não podem ser negligenciados, porque as condições de saúde são valores comunitários e determinados por aspectos ambientais e relacionados com a capacidade de consumo da população.

Além disso, o PBF configura-se apenas em uma peça de um sistema de direitos sociais básicos e está longe de ser considerado a panaceia das iniquidades, pois se acredita que a transferência de renda deva ser uma condição transitória ao passo que outros setores do estado devam agir de forma integrada, a fim de quebrar o ciclo de pobreza intergeracional, ofertando condições adequadas de educação, saúde e desenvolvimento a sua população.

No entanto, se o Bolsa Família não é a solução para todas as desigualdades, ao menos se apresenta como um meio capaz de contribuir para que elas se atenuem. Por fim, vale lembrar que desenvolvimento econômico também se faz com desenvolvimento social e políticas de assistência aos mais vulneráveis. Como provocação aos detratores do $\mathrm{PBF}$, vale também lembrar, evocando um antigo ditado popular, que se 'saco vazio não para em pé', um país com fome e doente estará fadado a desmoronar com seus altos índices de mortalidade infantil associada à desnutrição e à falta de assistência integral à saúde.

\section{Colaboradores}

Santos R (0000-0003-3345-1804)* e Bottega CG (0000-0001-6933-8120)* contribuíram igualmente na elaboração do manuscrito. 


\section{Referências}

1. Monteiro CA. A dimensão da pobreza, da desnutrição e da fome no Brasil. Estud. Avanç. 2003; 17(48):7-20.

2. Monteiro CA, Benicio MHD, Konno SC, et al. Causas do declínio da desnutrição infantil no Brasil, 19962007. Rev. Saúde Públ., 2009;43(1):35-43.

3. Horton R. Maternal and child undernutrition: an urgent opportunity. The Lancet. 2008; 371(9608):177274.

4. Caulfield LE, Richard SA, Rivera SA, et al. Stunting, wasting, and micronutrient deficiency disorders. 2006.

5. World Health Organization. The world health report 2008: primary health care: now more than ever. Genebra: WHO; 2008.

6. Black RE, Allen LH, Bhutta ZA, et al. Maternal and child undernutrition: global and regional exposures and health consequences. The Lancet. 2008; 371(9608):243-260.

7. Caulfield LE, Onis M, Blössner M, et al. Undernutrition as an underlying cause of child deaths associated with diarrhea, pneumonia, malaria, and measles. The American journal of clinical nutrition, 2004; 80(1):193-198.

8. Ramachandran P, Gopalan HS. Undernutrition \& risk of infections in preschool children. Indian Journal of Medical Research. 2009; 130(5):579-83.

9. Rede Interagencial de Informação para a Saúde. Indicadores básicos para a saúde no Brasil: conceitos e aplicações. 2. ed. Brasília, DF: Organização Pan-Americana da Saúde; 2008.

10. Brasil. Lei Federal no 10.836 , de 9 de janeiro de 2004 . Cria o Programa Bolsa Família e dá outras providências. Diário Oficial da União. 10 Jan 2004.

11. Brasil. Decreto ${ }^{\circ} 5.209$, de 17 de setembro de 2004 . Regulamenta a Lei $\mathrm{n}^{\circ}$ 10.836, de 9 de janeiro de 2004, que cria o Programa Bolsa Família, e dá outras providências. Diário Oficial da União. 18 Set 2004.

12. Instituto Brasileiro de Geografia e Estatística. Pesquisa Nacional por Amostra de Domicílios: segurança alimentar 2004/2009 [internet]. Rio de Janeiro: IBGE; 2010 [acesso em 2019 jun 10]. Disponível em: https://biblioteca.ibge.gov.br/index.php/biblioteca- catalogo?view=detalhes\&id=247241.

13. Stevenson WJ. Estatística aplicada à administração. São Paulo: Harbra: 2001.

14. Figueiredo Filho DB, Silva Júnior JA. Desvendando os mistérios do Coeficiente de Correlação de Pearson (r). Rev. Política Hoje. 2010; 18(1):115-146.

15. Ximenes DA, Agatte JP. A gestão das condicionalidades do Programa Bolsa Família: uma experiência intersetorial e federativa. Inclusão Soc. 2012; 5(1):1119.

16. Da Silva GA, Volpato LMB. Articulação entre a Política de Saúde e a Política de Assistência Social no âmbito do Programa de Transferência de Renda Bolsa Família, na busca de atender a totalidade das famílias beneficiárias [internet]. Seminário Integrado. 2015 [acesso em 2016 nov 15]; 9(9):1-19. Disponível em: http://intertemas.toledoprudente.edu.br/index.php/ SemIntegrado/article/view/5153/4905.

17. Silva MOS. Condicionalidades do Programa Bolsa Família. In: Rosa MCF, Hellmann A, organizadoras. Dicionário crítico: política de assistência social no Brasil. Porto Alegre: UFRGS, 2016. p. 56-59.

18. Ranganathan M, Lagarde M. Promoting healthy behaviours and improving health outcomes in low and middle income countries: a review of the impact of conditional cash transfer programmes. Preventive medicine. 2012; 55(suppl):S95-S105.

19. Brasil. Portaria $\mathrm{n}^{\circ} 325 / \mathrm{GM}$ de 21 de fevereiro de 2008. Estabelece prioridades, objetivos e metas do Pacto pela Vida para 2008, os indicadores de monitoramen- 
to e avaliação do Pacto pela Saúde e as orientações, prazos e diretrizes para a sua pactuação [internet]. [acesso em 2016 abr 23]. Disponível em: http://www. rio.rj.gov.br/dl static/10112/5127 629/41327 82/Port arian.325GMde21defevereirode2008.pdf.

20. Rasella D. Impacto do Programa Bolsa Família e seu efeito conjunto com a Estratégia Saúde da Família sobre a mortalidade no Brasil [tese]. Salvador: Instituto de Saúde Coletiva, Universidade Federal da Bahia; 2013. 92 p.

21. Bisquerra R, Sarriera JC, Matínez F. Introdução à estatística: enfoque informático com o pacote estatístico SPSS. Porto Alegre: Bookman, 2009.
22. Facchini LA, Piccini RX, Tomasi E, et al. Desempenho do PSF no Sul e no Nordeste do Brasil: avaliação institucional e epidemiológica da Atenção Básica à Saúde. Ciênc. Saúde Colet. v. 11, p. 669-681, 2006.

23. Asher HB. Causal modeling: Quantitative applications in the social sciences. Beverly Hills: Sage Publications, 1983.

Recebido em 14/01/2019

Aprovado em 18/07/2019

Conflito de interesses: inexistente

Suporte financeiro: não houve 